\title{
Efficacy and safety of TAS-102 in refractory metastatic colorectal cancer: a meta-analysis
}

This article was published in the following Dove Press journal:

Cancer Management and Research

\section{Duke Chen ${ }^{1, *}$ \\ Yu-Shen $\mathrm{Wu}^{2, *}$ \\ Huapeng $\operatorname{Lin}^{3, *}$ \\ Yihan Wang' \\ Longhao $\mathrm{Li}^{\prime}$ \\ Tao Zhang'}

'Department of Oncology, The First Affiliated Hospital of Chongqing

Medical University, Chongqing,

People's Republic of China;

${ }^{2}$ Chongqing Key Laboratory of

Molecular Oncology and Epigenetics,

The First Affiliated Hospital of

Chongqing Medical University,

Chongqing, People's Republic of

China; ${ }^{3}$ Department of Hepatobiliary

Surgery, The Second Affiliated

Hospital of Chongqing Medical

University, Chongqing, People's

Republic of China

*These authors contributed equally to this work
Correspondence: Longhao Li; Tao Zhang Department of Oncology, The First Affiliated Hospital of Chongqing Medical University, Number I Youyi Road, Yuzhong District, Chongqing, 400000, People's Republic of China Email lilonghaoon@163.com; tumorzt@163.com
Background: TAS-102 has been applied to metastatic colorectal cancer (mCRC) patients who had received at least two prior regimens of standard chemotherapy. This meta-analysis is designed to assess the efficacy and safety of TAS-102 in patients with mCRC.

Methods: We searched randomized controlled trials (RCTs) through PubMed, Embase, Web of Science and Cochrane clinical trial databases and clinicaltrial.gov from database initiation to March 2018. The overall survival (OS), progression-free survival (PFS), disease control rate (DCR) and incidence of adverse events were summarized with the use of hazard ratio (HR) or risk ratio (RR).

Results: Three RCTs with 1318 patients were included. Results showed that TAS-102 significantly improved OS (HR 0.70, 95\% confidence interval [CI] 0.62-0.79) and PFS (HR 0.46, 95\% CI $0.40-0.52$ ) in patients who were intolerant or refractory to fluoropyrimidine, irinotecan and oxaliplatin. The pooled odds ratio of DCR was 4.15 (95\% CI 3.18-5.43). Notably, there were significant OS benefits both in patients with KRAS mutation (HR 0.76, 95\% CI 0.63-0.92) and those with wild-type KRAS (HR 0.66, 95\% CI 0.55-0.79). These benefits were also observed in patients with different numbers of metastatic sites. However, patients with $>18$ months since the diagnosis of first metastases seemed to have better OS (HR $0.65,95 \%$ CI $0.55-0.77$ ). The most common toxicities associated with TAS-102 were neutropenia (RR 116.51, 95\% CI 23.51-577.33), leucopenia (RR 67.70, 95\% CI 13.63-336.29), anemia (RR 4.28, 95\% CI 2.70-6.79) and diarrhea (RR 5.10, 95\% CI 1.40-18.61).

Conclusion: TAS-102 significantly improves OS, PFS and DCR in refractory mCRC patients with tolerable toxicity. Meanwhile, the OS benefits have nothing to do with KRAS status and the number of metastatic sites.

Keywords: TAS-102, metastatic colorectal cancer, meta-analysis

\section{Introduction}

Colorectal cancer (CRC) is the third most frequent cancer and the fourth most frequent cause of cancer-related death worldwide. ${ }^{1,2}$ Approximately $25 \%$ of patients undergo metastases at initial diagnosis, and almost $50 \%$ of patients with CRC will ultimately develop metastases. ${ }^{3}$ With the arrival of the era of molecular targeted therapy, the median survival time of patients with metastatic colorectal cancer (mCRC) has been extended to 30 months. ${ }^{4-6}$ However, most patients with $\mathrm{mCRC}$ have to receive palliative care due to the fact that no more medicine is available after they are ultimately refractory or intolerant to standard chemotherapies (fluoropyrimidine, irinotecan and oxaliplatin). 
TAS-102, an oral antimetabolite, consists of two components: trifluridine (FTD) and tipiracil hydrochloride (TPI). ${ }^{7}$ FTD is a thymidine analog that plays a radical role in cytotoxicity through inhibiting thymidylate synthase (TS) and incorporation into DNA, resulting in DNA dysfunction and damage. ${ }^{8}$ TPI inhibits the rapid catabolism of FTD by thymidine phosphorylase (TP) at the first pass by the liver and the intestinal tract. ${ }^{9}$ Moreover, a review considers that TP is analogous to platelet-derived endothelial cell growth factor and TPI has a potential antiangiogenic activity. ${ }^{10}$

A Phase II clinical trial from Japan showed that TAS-102 significantly improved overall survival (OS) compared with placebo in mCRC patients who were refractory or intolerant to standard chemotherapies including 5-fluorouracil, irinotecan and oxaliplatin (OS 9.0, vs 6.6 months; hazard ratio [HR] $0.56,95 \%$ confidence interval [CI] 0.39-0.81); TAS-102 was therefore approved for treatment in $2014 .{ }^{11}$ The subsequent RECOURSE trial was a randomized, double-blind, placebocontrolled, international Phase III trial. ${ }^{12}$ It also demonstrated that TAS-102 had prominent efficacy in improving survival with tolerable adverse effects in refractory mCRC patients who had already received all approved cytotoxic or molecular targeted agents (OS 7.1, vs 5.3 months, HR 0.68, 95\% CI 0.58-0.81), which promote the approval of TAS-102 by the US Food and Drug Administration (FDA) in 2015. ${ }^{13}$

In this study, we aimed to perform meta-analysis of randomized controlled trials (RCTs) to further evaluate the efficacy and safety of TAS-102 in patients with mCRC.

\section{Materials and methods}

\section{Literature search and inclusion criteria}

We conducted literature search of RCTs on various databases including PubMed, Embase, Web of Science and Cochrane, as well as clinicaltrial.gov. We used the following keywords: "TAS-102", "Trifluridine", "chemotherapy" and "metastatic colorectal cancer", according to diverse combinations. Search time was from database initiation to March 2018, and there were no language restrictions.

The inclusion criteria were determined on the basis of "PICOS" principle: P, population: patients with mCRC; I, intervention: TAS-102 alone; $\mathrm{C}$, comparison: chemotherapy or placebo alone; $\mathrm{O}$, outcome: effect and safety and S, study: randomized Phase II or III trial. We selected trials by viewing titles and abstracts first. When we had uncertainties, the full texts would be read to rule out interference of the improper literature. Two investigators independently accomplished the search and review of all identified trials.

\section{Data extraction and quality assessment}

Two investigators independently extracted data from the included articles, including publication year, the primary end point, geographic region, characteristics of enrolled patients, sample sizes, study design and outcomes of different subgroups. The outcomes comprised OS, progressionfree survival (PFS), disease control rate (DCR) and adverse events. The Cochrane Collaboration's tool was applied for investigators to assess the quality of selected literature. The risk of bias assessment was measured by three forms for every trial: low risk (+), unclear risk (?) and high risk (-). When there were disagreements in all the processes, discussion and consultation with the third researcher were necessary to attain consistency.

\section{Data analyses and statistical methods}

Data analyses were performed using the RevMan 5.3 software (Cochrane Collaboration, Oxford, UK). OS and PFS, as the time-to-event results, were pooled through the most appropriate statistic, HRs. Subgroup analyses were used to appraising the consequences in patients with different KRAS statuses, times since the diagnosis of first metastases and numbers of metastatic sites. The incidence of adverse events was assessed through risk ratio (RR). All data were extracted directly from the reports, and the $95 \%$ CIs for all results were reported. As the RevMan software only retains two decimal places, when the $95 \%$ CIs were slightly different $(<0.01 \%)$ in statistical software and literature reports, we reported the former. Heterogeneity was evaluated through $\chi^{2}$-based $Q$ test and $I^{2}$ statistics. $I^{2}=0 \%$ expresses no heterogeneity is viewed, and a larger value means a larger heterogeneity. ${ }^{14}$ On account of the small number of included trials $(<10)$, we did not use the Begg's and Egger's trials to examine potential publication bias.

\section{Results}

\section{Included studies and study quality}

Initially, 377 articles were retrieved from all searched databases. We eliminated duplicates, reviews and single-arm trials by reading abstracts and then screened the full texts and excluded articles according to our inclusion criteria. Finally, three RCTs with 1318 patients were included in this study (Figure 1). The key baseline characteristics and the efficacy results of three included RCTs are summarized in Tables 1 and 2, respectively. All studies were randomized and double blind and reported all preset results, so the risk of bias was low by our judgment (Figure 2). 

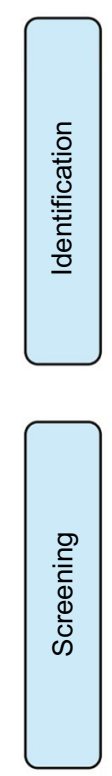

$$
\begin{aligned}
& \text { Records after duplicates removed } \\
& \qquad(\mathrm{N}=\mid 25)
\end{aligned}
$$
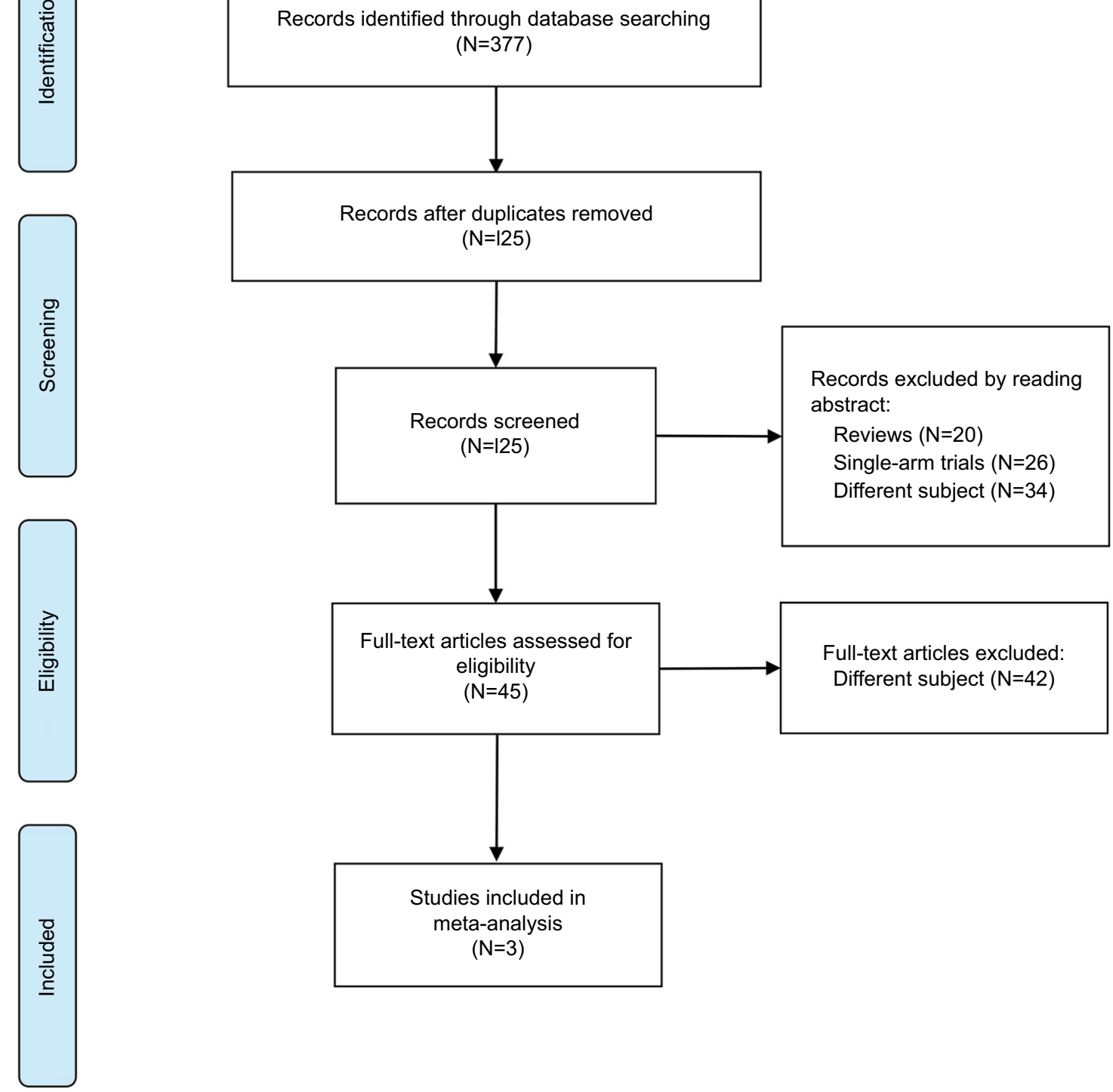

Figure I The process of study selection.

\section{OS}

OS was the primary end point of three trials, and the pooled HR showed that TAS-102 decreased the risk of death by $30 \%$ compared with placebo (HR $0.70,95 \%$ CI $0.62-0.79$, $I^{2}=24 \%$; Figure 3 ). The subgroup analyses were performed. Remarkably, TAS-102 had statistically significant OS benefits in patients with both KRAS mutation (HR 0.76, 95\% CI $0.63-0.92, I^{2}=44 \%$ ) and wild-type KRAS (HR 0.66, 95\% CI $0.55-0.79, I^{2}=2 \%$; Figure 4$)$. TAS- 102 prolonged OS in patients whether with one or two metastatic sites (HR 0.75 , $95 \%$ CI $0.62-0.90, I^{2}=20 \%$ ) or more than three metastatic sites (HR $0.67,95 \%$ CI $0.55-0.83, I^{2}=0 \%$ ). Interestingly, patients with $>18$ months since diagnosis of the first metastasis had OS improvement (HR 0.65, 95\% CI 0.55-0.77, $I^{2}=0 \%$ ), but the benefit was not observed in patients with $<18$ months since diagnosis of the first metastasis (HR 0.85 , $95 \%$ CI $\left.0.66-1.11, I^{2}=0 \%\right)$.

\section{Progression-free survival (PFS)}

PFS was significantly improved in patients who were treated with TAS- 102 (HR $0.46,95 \%$ CI $0.40-0.52, I^{2}=0 \%$; Figure 5). No more relevant data were recorded about PFS in the subgroup patients, so we could not perform deeper subgroup analysis. 


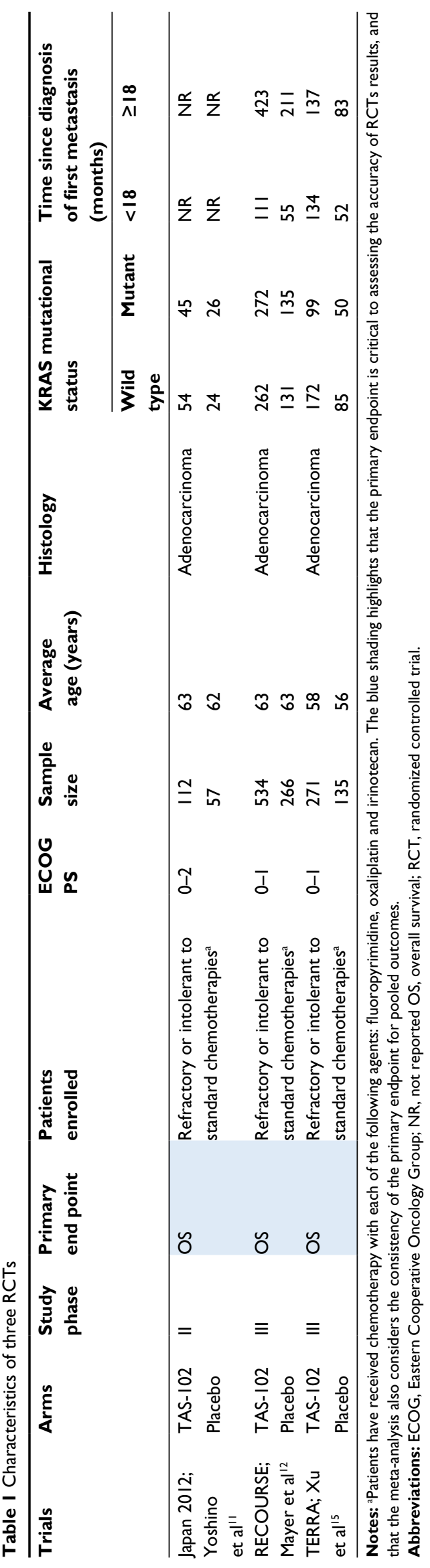

\section{DCR}

Based on the published DCR in three trials, the pooled odds ratio of DCR was 4.15 (95\% CI 3.18-5.43, $I^{2}=0 \%$; Figure 6). This result indicated the superiority of TAS-102 in improving DCR compared with placebo.

\section{Adverse events}

The most frequent grade 3 or higher adverse events associated with TAS-102 had been recorded in Figure 7. Consistent with previous reports, the application of TAS-102 would strikingly induce adverse events, including neutropenia (RR 116.51, 95\% CI 23.51-577.33, $I^{2}=0 \%$ ), leucopenia (RR 67.70, 95\% CI 13.63-336.29, $P=0 \%$ ), anemia (RR 4.28, 95\% CI 2.70-6.79, $I^{2}=3 \%$ ) and diarrhea (RR 5.10, 95\% CI $1.40-18.61, P=3 \%)$.

\section{Discussion}

As we know, TAS-102 had shown OS benefits in mCRC patients who were pretreated with fluoropyrimidine, irinotecan and oxaliplatin in previous two randomized clinical trials, giving rise to its approval in Japan, Europe and the USA. In the latest Phase III clinical trial (TERRA), TAS-102 still exhibited a statistically significant survival benefit compared with placebo in Asian patients with refractory mCRC (OS, 7.8; vs, 7.1 months; HR, 0.79; 95\% CI, 0.62-0.99) who were refractory or intolerant to two or more prior chemotherapy regimens, regardless of the previous exposure to targeted therapy..$^{15}$ In our study, the results of the three clinical trials were pooled and analyzed.

To our knowledge, this is the first meta-analysis to evaluate the efficacy and safety of TAS- 102 in patients with mCRC. The analysis showed that TAS-102 can significantly prolong OS and PFS in mCRC patients who had received at least two regimens of standard chemotherapy.

Notably, according to the outcomes of subgroup analyses, there was no significant difference of OS benefit in KRAS status and the number of metastatic sites. In the KRAS mutation subgroup, the reports in these three trials were different. Patients with wild-type KRAS had OS benefits only in the RECOURSE study, while KRAS-mutant patients had OS benefits only in the Japanese trial. In the Japanese trial, patients in the KRAS mutation group had superior OS than those in the KRAS wild-type group. The researchers executed an adjusted analysis subsequently, and the result was similar to the previous study. This discrepancy was eventually considered to be caused by the small sample size. In our study, the data from three clinical studies were accumulated and pooled, and it was concluded that the efficacy of TAS-102 was not 
Table 2 Efficacy results of three RCTs

\begin{tabular}{|c|c|c|c|c|c|c|c|c|c|c|}
\hline \multirow[t]{2}{*}{ References } & \multirow[t]{2}{*}{ Arms } & \multirow{2}{*}{$\begin{array}{l}\text { Sample } \\
\text { size }\end{array}$} & \multirow{2}{*}{$\begin{array}{l}\text { Primary } \\
\text { end point }\end{array}$} & \multicolumn{3}{|l|}{ OS } & \multicolumn{3}{|l|}{ PFS } & \multirow[t]{2}{*}{ DCR (\%) } \\
\hline & & & & $\begin{array}{l}\text { Median } \\
\text { (months) }\end{array}$ & HR & $95 \% \mathrm{Cl}$ & $\begin{array}{l}\text { Median } \\
\text { (months) }\end{array}$ & HR & $95 \% \mathrm{Cl}$ & \\
\hline Japan 20I2; & TAS-102 & 112 & OS & 9 & 0.56 & $0.39-0.81$ & 2 & 0.41 & $0.28-0.59$ & 43 \\
\hline Yoshino et al" & Placebo & 57 & & 6.6 & & & I & & & \\
\hline RECOURSE; & TAS- 102 & 534 & OS & 7.1 & 0.68 & $0.58-0.81$ & 2 & 0.48 & $0.4 \mathrm{I}-0.57$ & 44 \\
\hline Mayer et al ${ }^{12}$ & Placebo & 266 & & 5.3 & & & 1.7 & & & \\
\hline TERRA; Xu & TAS-I02 & 271 & OS & 7.8 & 0.79 & $0.62-0.99$ & 2 & 0.43 & $0.34-0.54$ & 44.1 \\
\hline et $\mathrm{al}^{15}$ & Placebo & 135 & & 7.1 & & & 1.8 & & & \\
\hline
\end{tabular}

Note: The blue shading highlights that the primary endpoint is critical to assessing the accuracy of RCTs results, and that the meta-analysis also considers the consistency of the primary endpoint for pooled outcomes.

Abbreviations: $\mathrm{Cl}$, confidence interval; DCR, disease control rate; HR, hazard ratio; OS, overall survival; PFS, progression-free survival; RCT, randomized controlled trial.

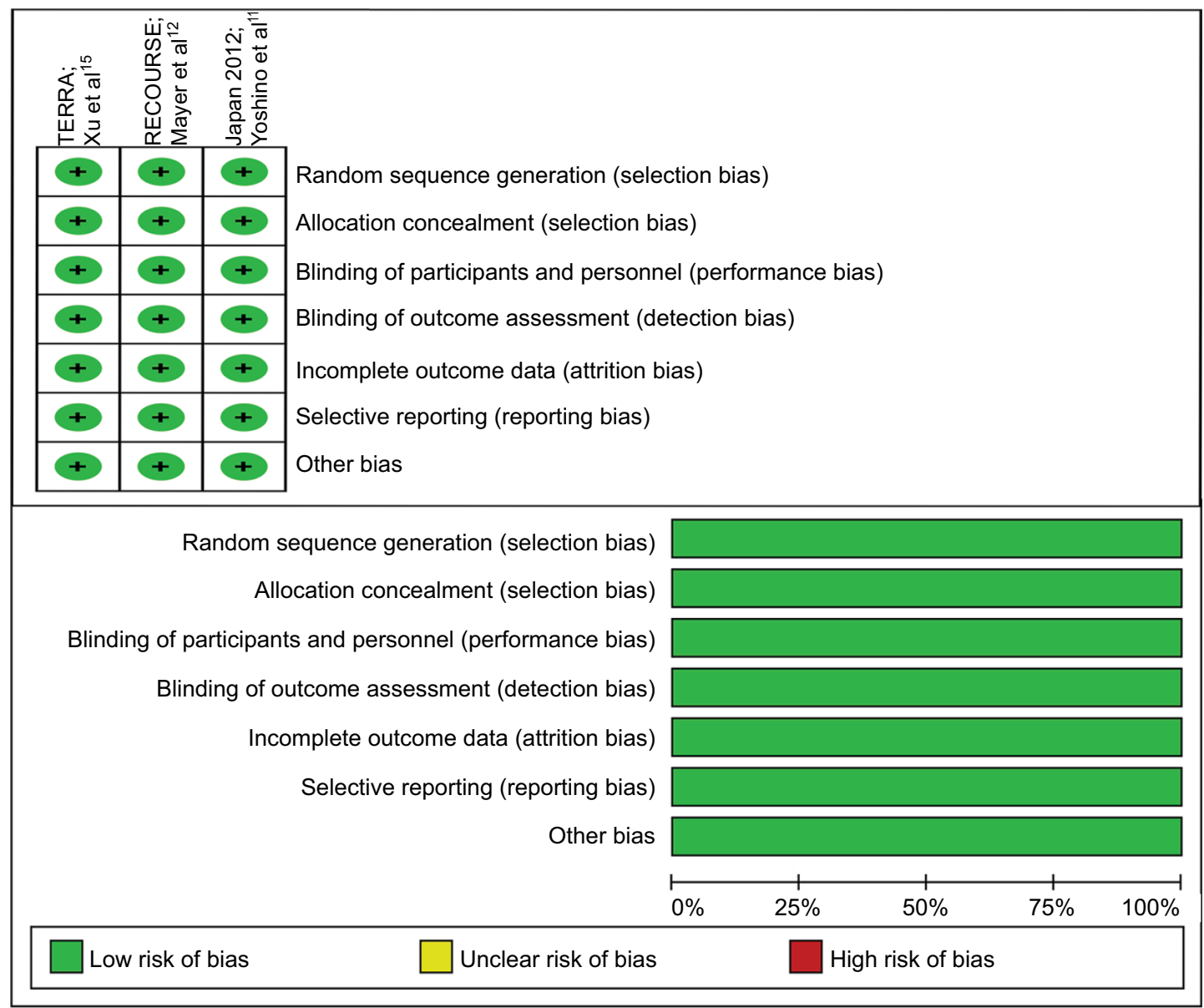

Figure 2 Risk of bias summary.

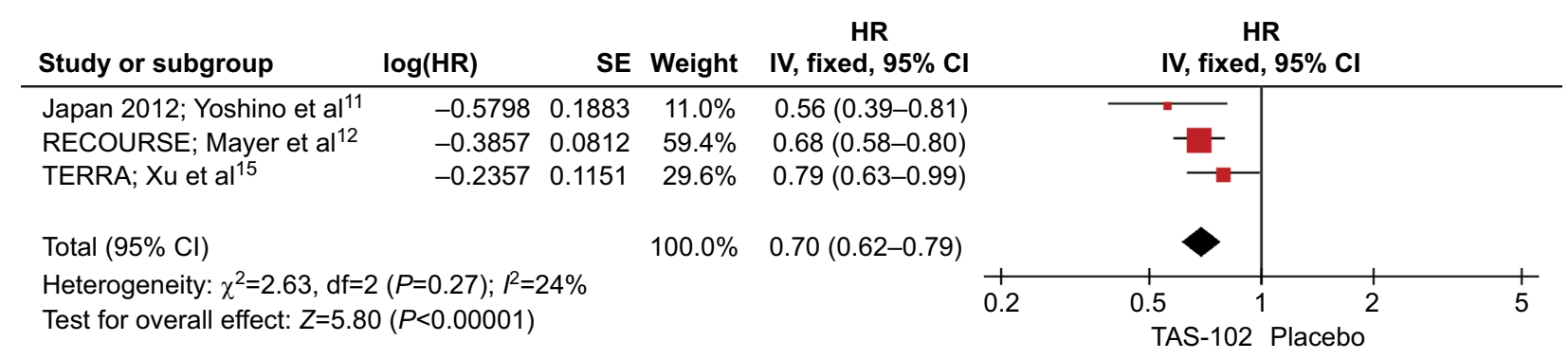

Figure 3 Forest plots for OS.

Abbreviations: $\mathrm{Cl}$, confidence interval; HR, hazard ratio; IV, inverse variance; OS, overall survival; SE, standard error. 


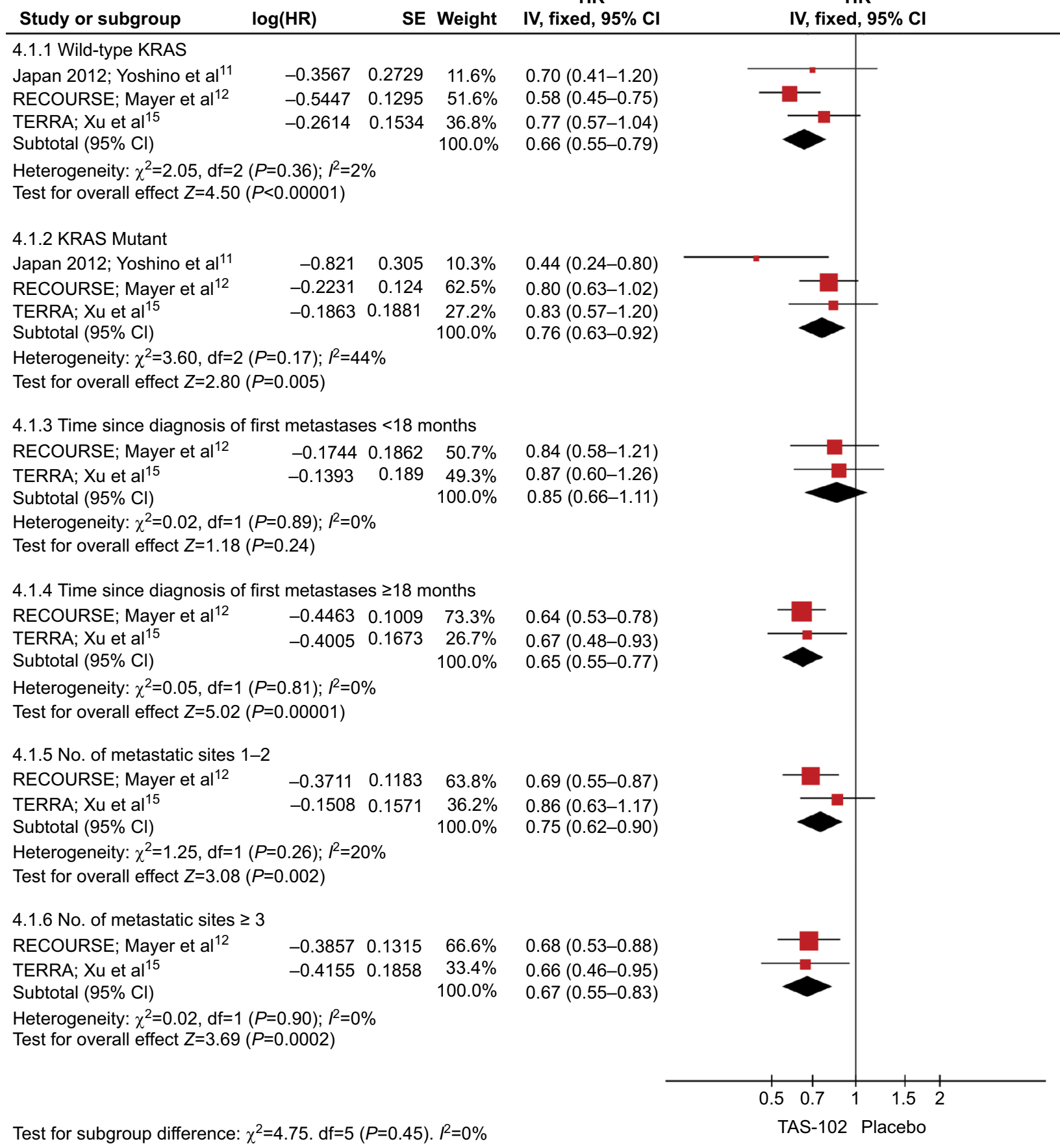

Figure 4 Forest plot for OS by subgroup.

Abbreviations: $\mathrm{Cl}$, confidence interval; HR, hazard ratio; IV, inverse variance; OS, overall survival; SE, standard error.

Study or subgroup

$\log (\mathrm{HR})$

Japan 2012; Yoshino et al ${ }^{11}$

RECOURSE; Mayer et al ${ }^{12}$

TERRA; Xu et al $^{15}$

Total $(95 \% \mathrm{Cl})$

Heterogeneity: $\chi^{2}=0.99, \mathrm{df}=2(P=0.61) ; P^{2}=0 \%$

Test for overall effect: $Z=12.58(P<0.00001)$
HR HR

SE Weight IV, fixed, $95 \% \mathrm{Cl} \quad$ IV, fixed, $95 \% \mathrm{CI}$

$0.41(0.28-0.59)$

$\begin{array}{lllll}-0.734 & 0.0804 & 60.0 \% & 0.48(0.41-0.56)\end{array}$

$\begin{array}{llll}-0.844 & 0.1162 & 28.7 \% & 0.43(0.34-0.54)\end{array}$

$100.0 \%$

$0.46(0.40-0.52)$

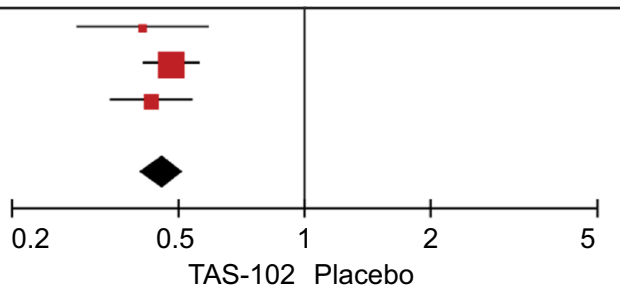

Figure 5 Forest plot for PFS.

Abbreviations: $\mathrm{Cl}$, confidence interval; HR, hazard ratio; IV, inverse variance; PFS, progression-free survival; SE, standard error. 
TAS-102 Placebo

Study or subgroup Events Total Events Total Weight $\mathrm{M}-\mathrm{H}$, fixed, 95\% $\mathrm{Cl}$

; Yoshino et al $^{11} 115 \quad 261$

RECOURSE; Mayer et al ${ }^{12} 221 \quad 534$

TERRA; Xu et al ${ }^{15} \quad 115 \quad 261$

Total $(95 \% \mathrm{Cl})$

Total events

1056

451

$\begin{array}{lll}19 & 130 & 23.2 \%\end{array}$

$42 \quad 266 \quad 53.7 \%$

$19 \quad 130 \quad 23.2 \%$

$4.60(2.67-7.93)$

$3.77(2.60-5.46)$

$4.60(2.67-7.93)$

$4.15(3.18-5.43)$

Heterogeneity: $\chi^{2}=0.54, \mathrm{df}=2(P=0.76) ; P^{2}=0 \%$

Test for overall effect: $Z=10.44(P<0.00001)$
OR

M-H, fixed, $95 \% \mathrm{Cl}$

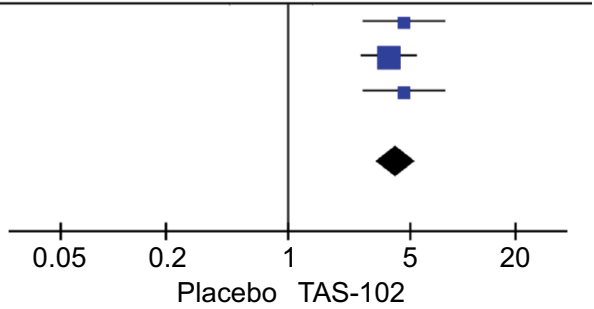

Figure 6 Forest plot for DCR.

Abbreviations: $\mathrm{Cl}$, confidence interval; $\mathrm{DCR}$, disease control rate; $\mathrm{M}-\mathrm{H}$, Mantel-Haenszel; OR, odds ratio.

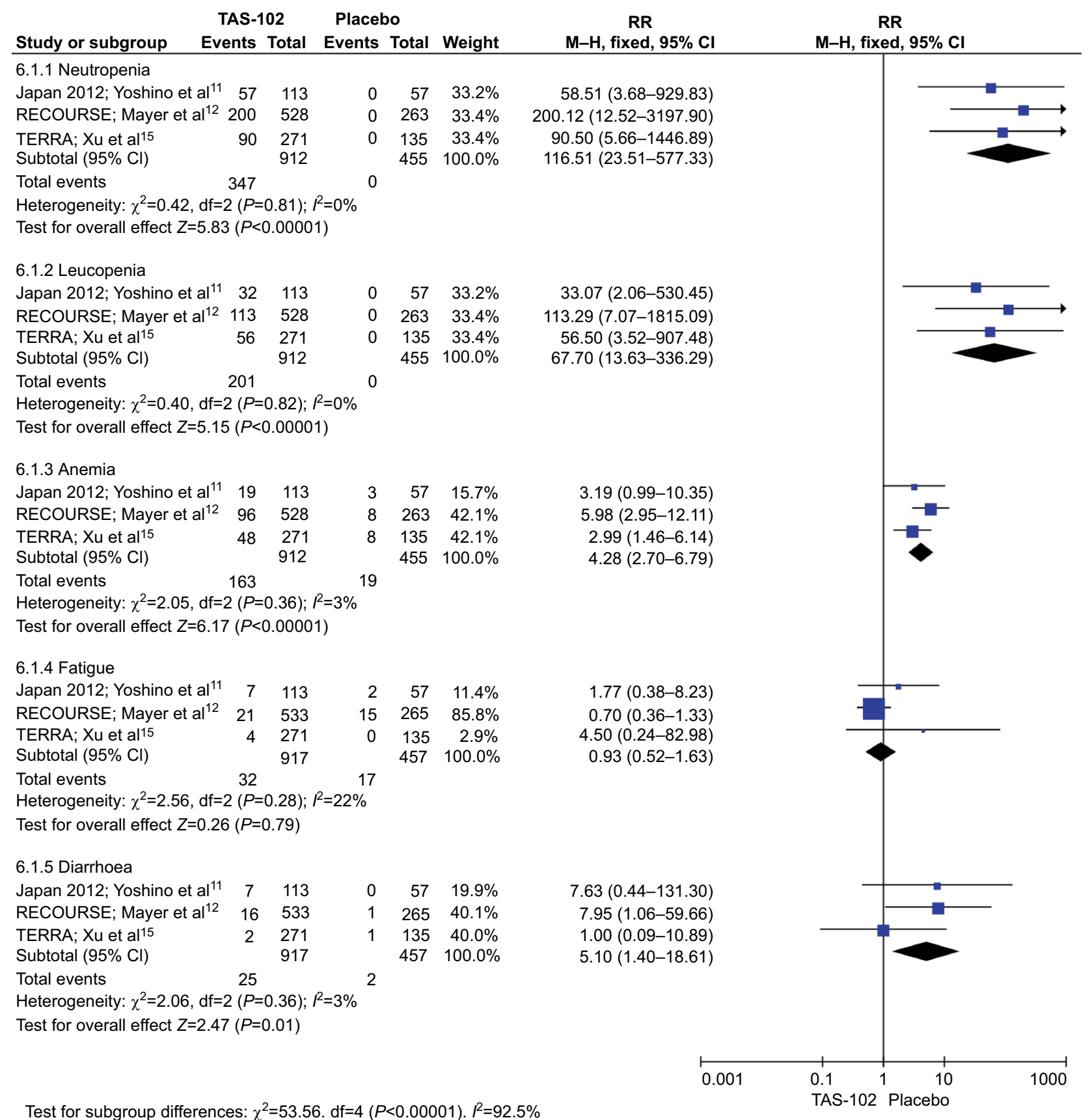

Figure 7 Forest plot for common grade 3 or higher adverse events.

Abbreviations: $\mathrm{Cl}$, confidence interval; $\mathrm{M}-\mathrm{H}$, Mantel-Haenszel; RR, risk ratio. 
related to KRAS status. The subgroup analyses demonstrated that TAS-102 seemed to be more effective in patients with $>18$ months since the diagnosis of first metastases, which may be related to the degree of malignancy of the tumor. However, these conclusions came from the subgroup analyses, which ought to be interpreted carefully. In consequence, further researches with more participants are requisite.

The most common adverse event induced by TAS-102 was myelosuppression. The frequent grade 3 or higher adverse events in the three trials are described as follows: neutropenia (33\%-50\%), leukopenia (21\%-28\%), anemia $(17 \%-18 \%)$, fatigue $(2 \%-6 \%)$ and diarrhea $(1 \%-6 \%)$. In these studies, at least one dose reduction occurred in $\sim 9 \%-20 \%$ of patients, and the withdrawal caused by adverse events took place in $4 \%-10 \%$ of patients who received TAS-102. Only one death in connection with TAS-102 was recorded, which was due to septic shock. TAS-102 was generally tolerated, and these adverse events could be adequately managed by reducing drug doses, prolonging chemotherapy intervals and giving related medicine. The common adverse events caused by TAS-102 are different from the toxicities of standard first-line chemotherapy regimens for mCRC. Gastrointestinal reactions and alopecia are more common in the FOLFIRI scheme, while more thrombocytopenia and neurologic toxicity are observed in FOLFOX4. ${ }^{16}$ This difference may provide a reference for the choice of additional treatment when patients are resistant to fluorouracil-based standard chemotherapy.

Although both TAS-102 and fluorouracil exert antitumor effects by inhibiting TS, the different molecular mechanisms between the two drugs may partly explain why TAS-102 is effective in patients refractory to fluorouracil. ${ }^{17}$ First, the routes of enzymatic catalysis pathway in DNA damage are not the same. The main cause of fluorouracil resistance is the decrease in orotate phosphoribosyltransferase (OPRT), a vital activated enzyme catalyzing fluorouracil to its monophosphate form, FdUMP, and an increased level of the target TS. Indeed, OPRT is not related to the mechanism of TAS-102. ${ }^{18}$ Second, autophagy limits the cytotoxicity of fluorouracil, resulting in resistance. However, FTD does not elicit autophagy and has more potential to cause cell death than 5-fluorouracil in a preclinical study. ${ }^{19}$ Third, FTD leads to the arrest of cell cycle in the G2/M phase, while fluorouracil arrests the cell cycle in the G1/S phase. ${ }^{20}$ Finally, TPI appears to have the ability of antiangiogenesis since TP is familiar with platelet-derived endothelial cell growth factor. The high expression of TP makes xenografted tumors more likely to relapse and metastasize. ${ }^{21}$
Similar to TAS-102, regorafenib has also shown a remarkable increase in OS with good tolerance in mCRC patients whose disease progresses after all available agents compared with placebo in two Phase III clinical trials, CORRECT and CONCUR. ${ }^{22,23}$ Based on these two studies, regorafenib is considered as an additional option for $\mathrm{MCRC}$ patients who still relapse after all standard treatments by the National Comprehensive Cancer Network and European Society for Medical Oncology guide. Since then, both regorafenib and TAS-102 are recommended as two novel choices for patients with intractable mCRC, due to these encouraging achievements. However, the optimal alternative in therapy management for refractory $\mathrm{mCRC}$ patients has not yet been determined. So far, no clinical studies directly compare the efficacy of the two drugs. In a network meta-analysis, investigators have made indirect comparisons and draw the conclusion that regorafenib and TAS-102 seem to have comparable ability to prolong survival with different toxicity profiles. ${ }^{24}$ This conclusion is in coincidence with results from some retrospective analysis. ${ }^{25,26}$ Furthermore, the best sequence of the two drugs is temporarily unreported. In the RECOURSE study, 144 patients who had been pretreated with regorafenib have parallel benefit on PFS in comparison with another 656 patients (HR 0.53, 95\% CI 0.36-0.78; vs HR 0.47, 95\% CI 0.39-0.56). However, some clinicians preferred to use regorafenib before TAS-102 when patients were generally in good conditions, because they considered that the first use of TAS-102 may worsen the patients' performance status on account of disease progression, which could prevent patients from being a candidate for regorafenib. ${ }^{27}$ After all, the purpose of treatment for advanced patients is to maximize the quality of life and extend life rather than shrinking the tumors. When the mCRC patients need an additional line of therapy, many factors should be taken into account, such as the purpose of the treatment, patient general conditions, previous chemotherapy regimens and drug toxicity characteristics. Besides, a Phase I study evaluating the safety and antitumor activity of TAS-102 administered in combination with regorafenib in $\mathrm{mCRC}$ patients who have progressed after standard therapy is recruiting (NCT03305913).

In addition, there are some clinical studies and preclinical studies investigating the efficacy and safety of TAS-102 in combination with other available drugs for mCRC. In two preclinical trials, the antitumor efficacy was significantly enhanced. The concentrations of FTD and FTD phosphates were obviously higher in combination treatment of bevacizumab and TAS-102 than either monotherapy on human CRC xenografts. ${ }^{28,29}$ Soon after, in order to assess effectiveness and security, an open-label, single-arm, multicenter 
Phase I/II study (C-TASK FORCE) was performed. ${ }^{30}$ A total of $25 \mathrm{mCRC}$ patients who were refractory or intolerant to standard treatments other than regorafenib were recruited in this study in Japan. The primary end point was 16 -week PFS, which finally reached $42.9 \%$ (80\% CI 27.8-59.0). The DCR was $64 \%$, and treatment-associated serious adverse events in patients, such as neutropenia, leukopenia and anemia, were $12 \%$. The results showed that TAS-102 together with bevacizumab had hopeful antineoplastic effects, which suggests that the combination treatment is an underlying alternative for refractory mCRC patients. Moreover, a Phase II study (TASCO1) assessing PFS in unresectable mCRC patients who are non-eligible for intensive therapy and receive TAS102 plus bevacizumab or capecitabine plus bevacizumab as first-line treatment is active (NCT02743221). Of course, except for bevacizumab, there are also studies that explore the combination management between TAS-102 and other targeting agents or cytotoxic drugs, such as cetuximab, panitumumab, oxaliplatin and irinotecan. ${ }^{31,32}$ These studies indicate that combination therapy is promising and provides the basis for further researches in mCRC.

In the future, we look forward to the possibility that there may be molecular markers that screen out the patients who can benefit from TAS-102, despite it is a cytotoxic drug. ${ }^{33}$ What is more, the combination of TAS-102 with other drugs and even radiotherapy may be a worthwhile treatment as a salvageable line in mCRC patients.

There are some limitations in this meta-analysis. In the first place, there were differences in the number of patients pretreated with targeted treatment among the three trials. Almost all patients received biologic treatment in RECOURSE, whereas only $45 \%$ of patients in the TAS- 102 arm and $51 \%$ of patients in the placebo arm were exposed to targeted therapy in TERRA. Second, the post-study treatments were various. Third, we pooled the results through HR and $95 \% \mathrm{CI}$ rather than individual patient data. Finally, the included three RCTs were inadequate to analyze sensitivity. However, this meta-analysis still provides high-quality evidence for the superiority of TAS-102 in mCRC patients.

\section{Conclusion}

TAS-102 plays a significant role in improving OS and PFS with a favorable safety profile in $\mathrm{MCRC}$ patients who are refractory or intolerant to standard treatment including fluorouracil, irinotecan, oxaliplatin, anti-VEGF and anti-EGFR. According to subgroup analysis results, these effects are not related to KRAS gene status and the number of metastatic sites. However, patients who have been $>18$ months since the diagnosis of first metastases seem to have survival benefits, which requires further researches to explore. In a word, TAS102 is a viable option in salvage therapy.

\section{Acknowledgments}

This study was supported by the Natural Science Foundation of Chongqing (number 2017MSXM005). Huapeng Lin is now affiliated with the Department of Intensive Care Unit, Affiliated Hangzhou First People's Hospital, Zhejiang University School of Medicine, Zhejiang, People's Republic of China.

\section{Disclosure}

The authors report no conflicts of interest in this work.

\section{References}

1. Ferlay J, Soerjomataram I, Dikshit R, et al. Cancer incidence and mortality worldwide: sources, methods and major patterns in GLOBOCAN 2012. Int J Cancer. 2015;136(5):E359-E386.

2. Arita S, Shirakawa T, Matsushita Y, et al. Efficacy and safety ofTAS-102 in clinical practice of salvage chemotherapy for metastatic colorectal cancer. Anticancer Res. 2016;36(4):1959-1966.

3. Van Cutsem E, Cervantes A, Nordlinger B, Arnold D, Group EGW. Metastatic colorectal cancer: ESMO clinical practice guidelines for diagnosis, treatment and follow-up. Ann Oncol. 2014;25(suppl 3):iii1-iii9.

4. Karapetis CS, Khambata-Ford S, Jonker DJ, et al. K-ras mutations and benefit from cetuximab in advanced colorectal cancer. $N$ Engl J Med. 2008;359(17):1757-1765.

5. Venook AP, Niedzwiecki D, Lenz HJ, et al. Cancer and leukemia group B (Alliance), SWOG, and ECOG: CALGB/SWOG 80405: phase III trial of FOLFIRI or mFOLFOX6 with bevacizumab or cetuximab for patients with KRAS wild-type untreated metastatic adenocarcinoma of the colon or rectum. J Clin Oncol. 2014;32:5s.

6. Chan DLH, Segelov E, Wong RS, et al. Epidermal growth factor receptor (EGFR) inhibitors for metastatic colorectal cancer (Review). Cochrane Database Syst Rev. 2017;6:CD007047.

7. van der Velden DL, Opdam FL, Opdam FL. TAS-102 and the quest for predictive biomarkers. ESMO open. 2017;2(4):e000263.

8. van der Velden DL, Opdam FL, Voest EE. TAS-102 for treatment of advanced colorectal cancers that are no longer responding to other therapies. Clin Cancer Res. 2016;22(12):2835-2839.

9. Suenaga M, Schirripa M, Cao S, et al. Potential role of polymorphisms in the transporter genes ENT1 and MATE1/OCT2 in predicting TAS-102 efficacy and toxicity in patients with refractory metastatic colorectal cancer. Eur J Cancer. 2017;86:197-206.

10. Temmink OH, Emura T, de Bruin M, Fukushima M, Peters GJ. Therapeutic potential of the dual-targeted TAS-102 formulation in the treatment of gastrointestinal malignancies. Cancer Sci. 2007;98(6):779-789.

11. Yoshino T, Mizunuma N, Yamazaki K, et al. TAS-102 monotherapy for pretreated metastatic colorectal cancer: a double-blind, randomised, placebo-controlled phase 2 trial. Lancet Oncol. 2012;13(10):993-1001.

12. Mayer RJ, Van Cutsem E, Falcone A, et al. Randomized trial of TAS-102 for refractory metastatic colorectal cancer. $N$ Engl J Med. 2015;372(20):1909-1919.

13. Marcus L, Lemery SJ, Khasar S, et al. FDA approval summary: TAS102. Clin Cancer Res. 2017;23(12):2924-2927.

14. Huedomedina TB, Sánchezmeca J, Marínmartínez F, Botella J. Assessing heterogeneity in meta-analysis: Q statistic or I2 index? Psychol Methods. 2006;11(2):193. 
15. Xu J, Kim TW, Shen L, et al. Results of a randomized, double-blind, placebo-controlled, phase III trial of trifluridine/tipiracil (TAS-102) monotherapy in Asian patients with previously treated metastatic colorectal cancer: the TERRA study. J Clin Oncol. 2018;36(4):350-358.

16. Colucci G, Gebbia V, Paoletti G, et al; Gruppo Oncologico Dell'Italia Meridionale. Phase III randomized trial of FOLFIRI versus FOLFOX4 in the treatment of advanced colorectal cancer: a multicenter study of the Gruppo Oncologico Dell'Italia Meridionale. J Clin Oncol. 2005;23(22):4866-4875.

17. Peters GJ, Bijnsdorp IV.TAS-102: more than an antimetabolite. Lancet Oncol. 2012;13(12):e518.

18. Lenz HJ, Stintzing S, Loupakis F. TAS-102, a novel antitumor agent: a review of the mechanism of action. Cancer Treat Rev. 2015;41(9):777-783.

19. Bijnsdorp IV, Peters GJ, Temmink OH, Fukushima M, Kruyt FA. Differential activation of cell death and autophagy results in an increased cytotoxic potential for trifluorothymidine compared to 5 -fluorouracil in colon cancer cells. Int J Cancer. 2010;126(10):2457-2468.

20. Matsuoka K, Iimori M, Niimi S, et al. Trifluridine induces p53-dependent sustained $\mathrm{G} 2$ phase arrest with its massive misincorporation into DNA and few DNA strand breaks. Mol Cancer Ther. 2015;14(4):1004-1013.

21. Bronckaers A, Gago F, Balzarini J, Liekens S. The dual role of thymidine phosphorylase in cancer development and chemotherapy. Med Res Rev. 2009;29(6):903-953.

22. Grothey A, Van Cutsem E, Sobrero A, et al; CORRECT Study Group. Regorafenib monotherapy for previously treated metastatic colorectal cancer (CORRECT): an international, multicentre, randomised, placebocontrolled, phase 3 trial. Lancet. 2013;381(9863):303-312.

23. Li J, Qin $\mathrm{S}, \mathrm{Xu} \mathrm{R}$, et al; CONCUR Investigators. Regorafenib plus best supportive care versus placebo plus best supportive care in Asian patients with previously treated metastatic colorectal cancer (CONCUR): a randomised, double-blind, placebo-controlled, phase 3 trial. Lancet Oncol. 2015;16(6):619-629.
24. Abrahao ABK, Ko YJ, Berry S, Chan KKW. A comparison of regorafenib and TAS-102 for metastatic colorectal cancer: a systematic review and network meta-analysis. Clin Colorectal Cancer. 2017;17(2):113-120.

25. Masuishi T, Taniguchi H, Hamauchi S, et al. Regorafenib versus trifluridine/tipiracil for refractory metastatic colorectal cancer: a retrospective comparison. Clin Colorectal Cancer. 2017;16(2):e15-e22.

26. Sueda T, Sakai D, Kudo T, et al. Efficacy and safety of regorafenib or TAS-102 in patients with metastatic colorectal cancer refractory to standard therapies. Anticancer Res. 2016;36(8):4299.

27. Grothey A, Marshall JL, Seery TE. Current options for third-line treatment of metastatic colorectal cancer. Clin Adv Hematol Oncol. 2016;14(3 suppl 3):1-15.

28. Ishida K, Sakamoto K, Tanaka N, et al. 22 Novel combination therapy, TAS-102 combined with the anti-EGFR antibody or the anti-VEGF antibody showed therapeutic benefit toward colorectal cancer xenografts. Eur J Cancer. 2014;50(6):13-13.

29. Tsukihara H, Nakagawa F, Sakamoto K, et al. Efficacy of combination chemotherapy using a novel oral chemotherapeutic agent, TAS-102, together with bevacizumab, cetuximab, or panitumumab on human colorectal cancer xenografts. Oncol Rep. 2015;33(5):2135-2142.

30. Kuboki Y, Nishina T, Shinozaki E, et al. TAS-102 plus bevacizumab for patients with metastatic colorectal cancer refractory to standard therapies (C-TASK FORCE): an investigator-initiated, open-label, single-arm, multicentre, phase 1/2 study. Lancet Oncol. 2017;18(9): $1172-1181$.

31. Temmink OH, Hoebe EK, Born KVD, Ackland SP, Fukushima M, Peters GJ. Mechanism of trifluorothymidine potentiation of oxaliplatin-induced cytotoxicity to colorectal cancer cells. Br J Cancer. 2007;96(2):231.

32. Baba Y, Tamura T, Satoh Y, et al. Panitumumab interaction with TAS2 leads to combinational anticancer effects via blocking of EGFR-mediated tumor response to trifluridine. Mol Oncol. 2017;11(8):1065-1077.

33. Burki TK. TAS-102 in metastatic colorectal cancer. Lancet Oncol. 2018;19(1):e18
Cancer Management and Research

\section{Publish your work in this journal}

Cancer Management and Research is an international, peer-reviewed open access journal focusing on cancer research and the optimal use of preventative and integrated treatment interventions to achieve improved outcomes, enhanced survival and quality of life for the cancer patient. The manuscript management system is completely online and includes

\section{Dovepress}

a very quick and fair peer-review system, which is all easy to use. Visit http://www.dovepress.com/testimonials.php to read real quotes from published authors. 